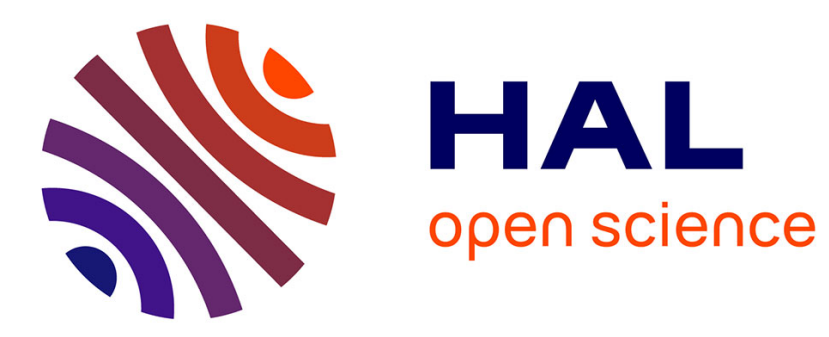

\title{
WiMAX-based Overlay Conferencing Service
}

\author{
Quoc T. Tran, Tien Anh Le, Hang Nguyen
}

\section{To cite this version:}

Quoc T. Tran, Tien Anh Le, Hang Nguyen. WiMAX-based Overlay Conferencing Service. 2011 15th International Conference on Intelligence in Next Generation Networks (ICIN) - From Bits to Data, From Pipes to Clouds (ICIN 2011), citeulike-article-id = 9318445, 2011, Berlin, Germany, Germany. pp.1-6. hal-00625859

\section{HAL Id: hal-00625859 \\ https://hal.science/hal-00625859}

Submitted on 22 Sep 2011

HAL is a multi-disciplinary open access archive for the deposit and dissemination of scientific research documents, whether they are published or not. The documents may come from teaching and research institutions in France or abroad, or from public or private research centers.
L'archive ouverte pluridisciplinaire HAL, est destinée au dépôt et à la diffusion de documents scientifiques de niveau recherche, publiés ou non, émanant des établissements d'enseignement et de recherche français ou étrangers, des laboratoires publics ou privés. 


\title{
WiMAX-based overlay conferencing service
}

\author{
Quoc Tuan Tran, Tien Anh Le, Hang Nguyen
}

\begin{abstract}
This paper proposes a new service architecture based on the interconnectivity between Subscriber Units (SUs) running on WiMAX network participating in an Application Layer Multicasting-based distributed multimedia conference. The novelty of the proposal is that it replaces the standard centralized architecture of IMS-based conference in order to utilize the intelligence and computational capacity of WiMAX Base stations. Thank to a distributed architecture, the expensive Media Resource Function Controller (MRFC) and Media Resource Function Processor (MRFP) can be completely omitted in this proposal. Experimental results show that the proposed architecture outperforms the previous system. Afterward, a prototype has been built to measure and prove the advanced performance of the proposed architecture.
\end{abstract}

Index Terms - video conferencing service; distributed system; WiMAX; IMS;

\section{INTRODUCTION}

Video conference is the most complex type of service among variety of telecommunication services. It requires a guaranteed quality of service and a real-time interactivity. Nowadays, there are two main types of service architectures used by video conference services [1]: Centralized architecture using Multipoint Control Unit (MCU) and distributed architecture using multicast mechanisms. The centralized architecture has advantages such as an easy management of admission control and a popular architecture which is currently being widely supported, but its main disadvantages are an exponential cost if the number of participants is increased, and a single point of failure. Because of the scalability and load balancing, there is another solution: distributed architecture.

The mobile terminals are now equipped with a high computational capacity and a long battery life. New radio access technologies, such as LTE, WiMAX (in this research work, WiMAX network is used as a demonstration of the $4 \mathrm{G}$ infrastructure.), can enhance the mobile applications with a high throughput. Accordingly, these mobile terminals can participate into a distributed conference session together with desktop or laptop computers.

The main problem is that the distributed video conferencing service and $4 \mathrm{G}$ network architecture have been designed with different requirements. The $4 \mathrm{G}$ network is based on the IP Multimedia Subsystem (IMS) to provide multimedia services including video conferencing service. However, the IMS-based video conference relies on a centralized service architecture or MCU [2]. Hence, it has same problems with centralized video conference. For example, when the number of UEs increases, the centralized architecture will sustain a huge load for mixing and distributing video data. In this research work, we propose an IMS based WiMAX infrastructure which can seamlessly support the distributed video conferencing service. The main contributions of this research is that we propose an IMS-based architecture service without applying a central-

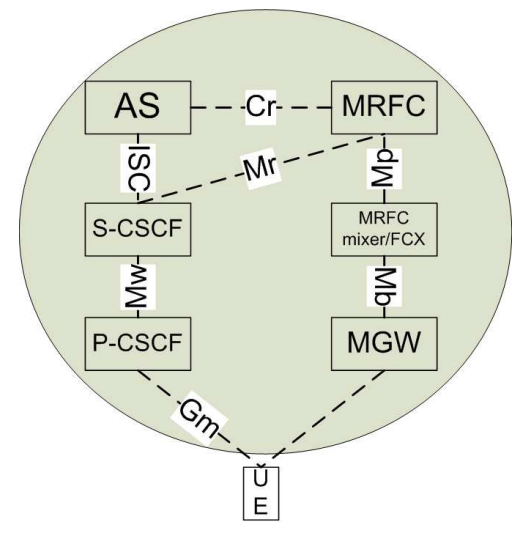

Fig. 1. 3GPPs IMS-based centralized conference architecture.

ized MCU using the interconnectivity between the IMSWiMAX platforn and WiMAX Subscriber Users (SU) participating in the distributed conference.

The rest of this paper is organized as follows. First, we briefly describe the background knowledge including: IMSbased standard for video conferencing, WiMAX and interconnectivity between IMS and WiMAX, and the ALM architecture. Then, we present an IMS-based video conferencing service applying a distributed architecture with common operations such as: join/leave, pause/return, hand-over.

\section{BACKGROUND KNOWLEDGE AND RELATED WORK}

\section{A. 3GPPs IMS-based Conferencing architecture}

Figure 1 presents the $3 \mathrm{GPP}$ standard for the IMS-based conference architecture. In this standard, there are two principal protocols: Session Initiation Protocol (SIP) and Real-time Transfer Protocol (RTP) which are signaling and media transport protocols. The IMS architecture uses a media gateway (MGW) for transmitting and forwarding media data to UE. In this conference standard, two important components are applied: Media Resource Function Controller (MRFC) and Application Server (AS). MRFC controls a small element which is Media Resource Function Processor. MRFC provides all media related functions required by a conference service such as mixing, transcoding, trans-rating. It may also have the Floor Control Server(FCS). Since all media related functions are controlled by the MRFC, this architecture is centralized. To avoid the disadvantages of the IMS-based conferencing standard, several proposals have been made. Their common purpose is to support the distributed conferencing service on current centralized architecture of IMS-based conferencing service with WiMAX access network. F.Belqasmi et. al. [2] proposed the FCS feature which is separated from MRFP. However, his solution did not change the centralized architecture of IMS-based conferencing standard. 


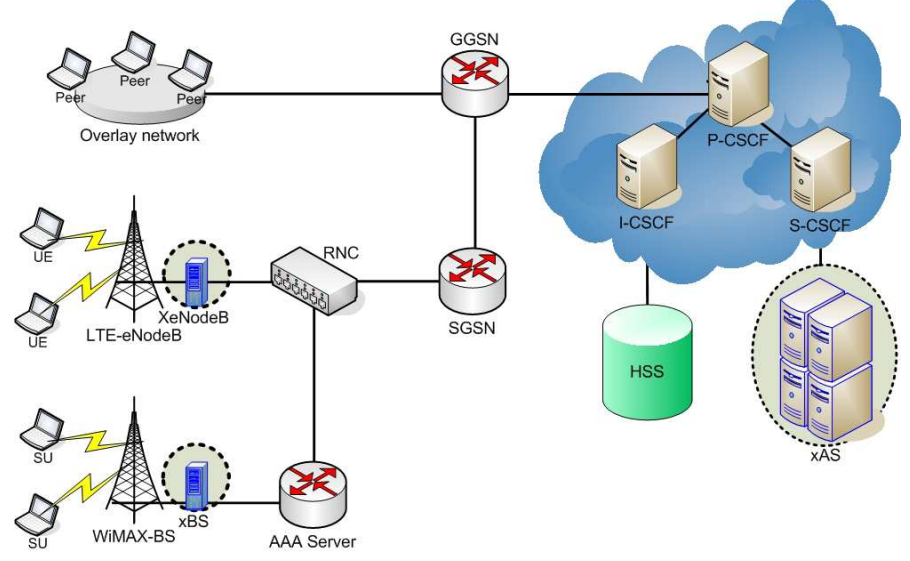

Fig. 2. Loose couple of interworking.

In [3], A.Buono et. al. proposed a distributed solution as an overlay network of centralized conferencing clouds. In addition, it has used a Content Distributed Network (CDN) with proxy servers to support distributed contents, but all participants still have to process all signaling and media loads. CDN servers are mainly proxy-based MCUs to connect some clients together using a centralized architecture and then connect other centralized groups together by creating an overlay network of those proxy servers. To conclude, the current solutions for IMS-based conferencing service either are centralized or have not fully utilized the capacity of the 4Gs infrastructure such as WiMAXs.

\section{B. WiMAX and the interconnectivity between WiMAX and IMS}

WiMAX is a broadband wireless access (BWA) technology for wireless metropolitan area networks. It was created by the WiMAX Forum, which was founded in June 2001 to promote the adoption of WiMAX compatible products and services [4]. A WiMAX network usually contains the following network entities [4]: ASN (Access Service Network), CSN (Connectivity Service Network), ASP (Access Service Provider) and MS/SS (Mobile station/Subscriber station). ASN is an entity which provides radio access to WiMAX subscriber, it also has some features such as: transferring of AAA authentication messages, authorization and session accounting for subscriber sessions, radio resource management.[4], CSN provides IP connectivity services to WiMAX subscribers meaning that CSN supplies several functions such as: Internet access, AAA proxy and server, Policy and Admission Control based on user subscriptions profiles [4] ASP is a business entity which provides applications or services [4]. In this entity, the WiMAX Forum proposes two types of connetion to an application or a service: nonIMS application server and P-CSCF(IMS). However, it is not clear how to develop a non-IMS application server [4]. Thus, we suppose that they use the IMS-based application server to provide services for WiMAX subscribers.

WiMAX has two types of inter-connection with other wireless networks such as LTE(3GPP): loose couple and tight couple[5]. Figure 2 shows that loose couple interwork-

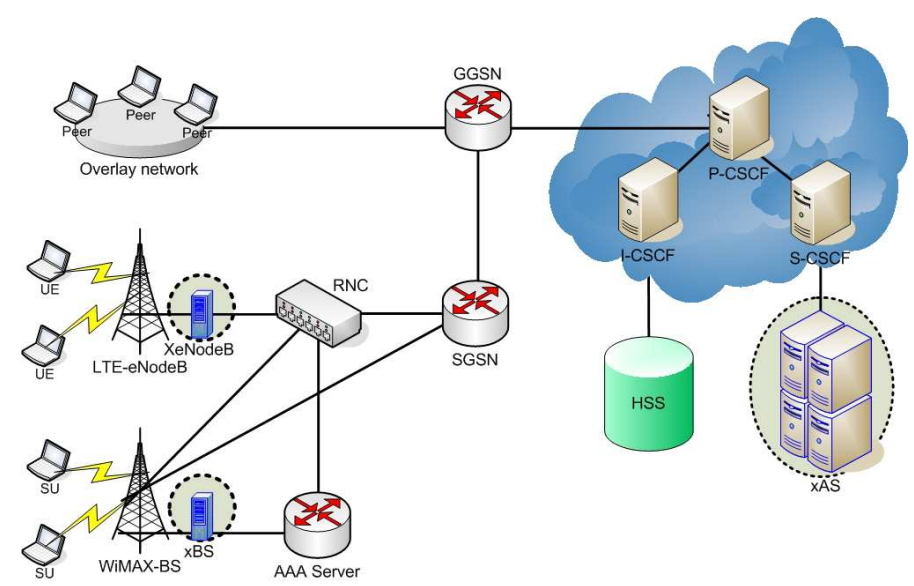

Fig. 3. Tight couple of interworking.

ing re-uses the AAA-server of $3 \mathrm{GPP}$ network. The core network (SGSN, GGSN) does not transfer data stream. This type has an advance: independence of WiMAX network. On the other hand, Fig.3 shows that tight couple uses RNC and core network (e.x : SGSN and GGSN). Therefore, there are some changes in its protocol, interface and service for interworking requirements. However, these two types of interworking provide the interconnectivity between WiMAX subscribers and the IMS platform or an IMS-based service. In fact, it is possible to provide services or applications for WiMAX subscribers via IMS.

\section{IMS-BASED DISTRIBUTED VIDEO CONFERENCING SERVICE FOR WIMAXS SUBSCRIBERS}

\section{A. Main challenges}

There are several challenges which are blocking IMSbased distributed video conferencing from providing services for WiMAXs subscribers. IMS is firstly a 3Gpps architecture for providing commercial services and applications regardless of the underlay network. On the other side, WiMAX is the standard proposed by the WiMAX Forum and is standardized to IEEE 802.16 [4]. It is therefore called a Non-3GPP standard. Accordingly, it is difficult to connect between WiMAX and IMS, especially as providing IMS-based video conferencing service to WiMAXs subscriber. However, in a draft standard [4], WiMAX Forum has presented a method to connect between WiMAX and IMS. Even though, IMS has proposed a standard for video conferencing service, it is still based on centralized architecture [2]. Therefore, our research work manage to discard components of the centralized architecture such as MRFC, MRFP in order to avoid bottle-neck phenomenon and to reduce the entire system's CAPEX/OPEX. ALM works on application layer, it does not care which kind of access network technology is used by the terminal for participating into a conference session. To maintain ALM operations such as heart-beat, data-forwarding, all members use their resources, for instance: bandwidth, memory, computational processor. It is difficult to apply ALM in mobile terminals. Moreover, WiMAX is a mobile network in which components connect together using the same core network. 


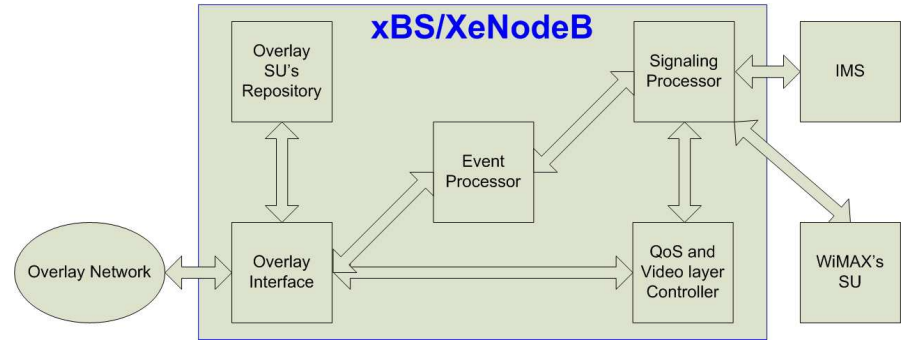

Fig. 4. Block diagram features of WiMAX extended base station.

WiMAX BSs are smart base stations, they are capable of computing and sharing loads[10]. If these WiMAX BSs can represent UEs in an ALM overlay network to forward their data streams and to process signaling messages, UEs can participate into a distributed conferencing session as if they are participating in the conventional IMS-based centralized conference. Next, a distributed video conferencing service using ALM mechanism does not have any method for managing floor control (methods to handle conferences such as participants' joint/leave, pause/return, select and manage the active users). Thus, our architecture proposes a floor control to support ALM-based distributed conference. Another important limitation is that, a distributed video conferencing service working on WiMAX does not support QoS. On the other side, WiMAX owns a mechanism for supporting QoS and so does IMS [4]. Therefore, a QoS converting mechanism between IMS and WiMAX is necessary to provide a QoS-guaranteed ALM conferencing service. Last but not least, our proposed architecture should support common mobile operations in a video conferencing session such as: join/leave, pause/return and hand-over.

\section{B. Extended WiMAX base station and extended applica- tion server for WiMAX proposal for IMS-based dis- tributed conferencing service}

To construct an IMS-based distributed conferencing service for WiMAXs subscribers, we propose two extended components: IMS-based Extended Application Server and Extended WiMAX Base Station. Extended IMS-based provides the principal signaling video conferencing service, it also has floor control features for video conferencing session. Extended WiMAX BS (WiMAX xBS) is representative of some SUs that it is managing in the overlay network. It means that WiMAX xBS should understand the interface between ALM architecture and WiMAX-IMS architecture. Figure 4 shows a block diagram featuring an extended WiMAX base station. In general, this extended WiMAX base station is representative of several nodes that it is managing in the Overlay network. At first, when a WiMAX SU wants to participate into the conference, they sends their signaling over messages such as REGISTER or INVITE SIP messages to the signaling processor in WiMAX BS. Furthermore, these messages contain QoS and Layer registration information indicating that a WiMAX SU wants to receive the video stream. The signal-

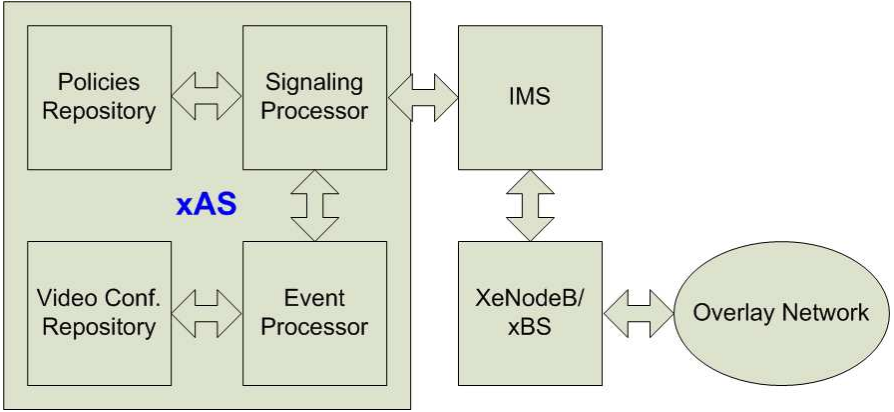

Fig. 5. Block diagram featuring an extended Application Server (xAS).

ing processor will handle this information to provide QoS and layer registration. Then QoS and layer registration demands the IMS core network to check the conditions of the WiMAXs SU such as: policies, bandwidth via Signaling processor. If WiMAXs SUs capacities are enough to receive scalable video, then QoS Layer notifies those requirements to the Overlay network via its Overlay Interface. Secondly, having received events in video conferencing session, the WiMAX SU sends these commands via SIP messages, the signaling processor sends commands to the event processor. Next, this event processor will handle all events in a video conference session. Thus, it may send requirements to the Overlay network via Overlay Interface. At the same time, WiMAX xBS will update SUs information to the Overlay SUs Repository such as: UEs IP, role of overlay network (source/relay/forwarding), status (idle/active), video layer registration. Figure 5 illustrates the block diagram feature of application server (AS). We propose this extended IMS-based Application Server (xAS). The principal features of xAS are floor control in video conferencing service and obtaining WiMAXs SUs information from the IMSWiMAX core network without requesting information from WiMAXs SUs. First of all, when receiving an event from the video conferencing session, WiMAX xBS sends several signaling messages to $\mathrm{xAS}$, and $\mathrm{xAS}$ receives these messages via its Signaling processor interface, processes events and then updates them to Video conferencing policy repository such as: conference ID, WiMAX BSs ID, status of conference (in process, paused or terminated). Thus, xAS can manage the floor control, for example: member joining, leaving, pausing and returning. On the other hand, because of the controlling process in Overlay network, all members have to transfer signaling messages. It is not practical for mobile terminals, because it takes too much of their resources such as: bandwidth, computing capacity, battery life. The xAS can obtain WiMAXs SU information using the core IMS-WiMAX network. After obtaining this information, xAS sends it to WiMAX xBS which is the representative of nodes it is managing. WiMAX xBS will convert the content and payload it to the signaling messages and send them to the Overlay network. This process helps saving mobile terminals resources by making use of WiMAX xBS capacity. Moreover, xAS assigns the policies for a video conferencing session such as: start time, du- 


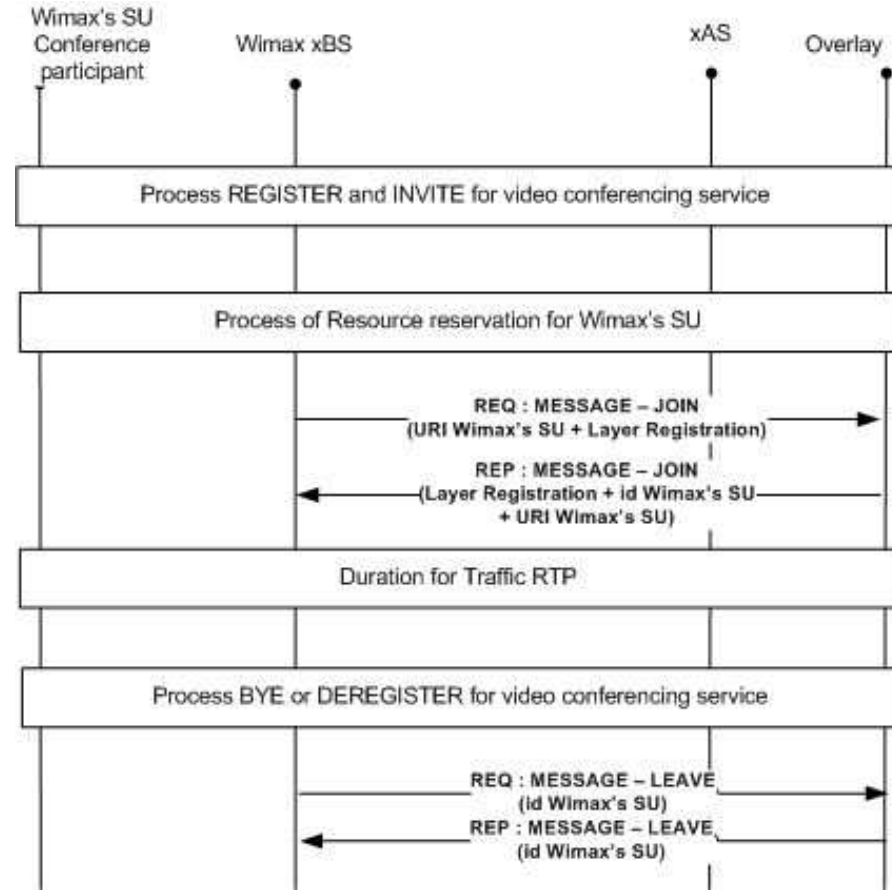

Fig. 6. Join/Leave of WiMAXs SU to/from distributed conference with QoS support.

ration, maximum number of participants, QoS parameters and Billing information. This information is stored in the Policies Repository.

\section{Common operations for a distributed conferencing ses- sion}

\section{C.1 JOIN/LEAVE OPERATION}

Figure 6 shows a call-flow when a WiMAX SU participates in or leaves a distributed video conference. First of all, when a WiMAX SU wants to use the video conferencing service, it may have to use the REGISTER and INVITE processes. It will automatically send the REGISTER message to its WiMAX xBS, then WiMAX xBS stores information about WiMAXs SU such as: UE-SIP-Account. Next WiMAX xBS transfers information of WiMAXs SU to xAS. After receiving this information, xAS updates the Video conferencing repository. To initiate its participation in a distributed conference, WiMAX SU sends the INVITE message. INVITE message contains information such as: Conference ID - identity for a conferencing session, Layer Registration - maximum number of enhancement layer(s) it wants to receive from the conference multicast tree. WiMAX xBS converts this layer requirements to QoS parameters which are understandable by the WiMAX-IMS core network. Afterwards, the xAS receives the INVITE message for mapping among: Conference ID, WiMAXs SU-SIP-Account, and WiMAX BS ID in the Video conferencing repository.

At the same time, IMS-WiMAX core network will check WiMAXs SU subscription whether it has enough participating rights and QoS resources in order to receive the required number of enhancement video layers. If its subscrip-

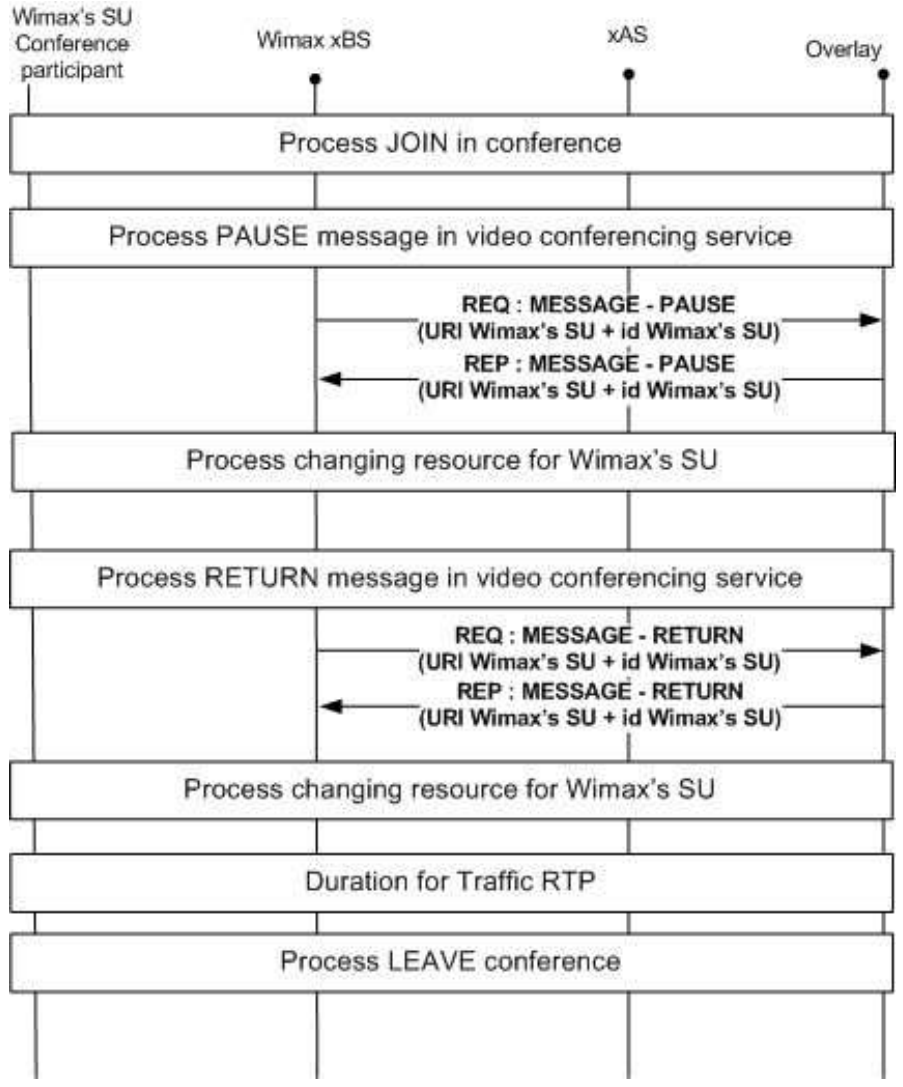

Fig. 7. Pause/Return operations over distributed video conferencing service with QoS support.

tion is enough, the IMS-WiMAX core work will reserve a resource in WiMAX xBS for WiMAXs SU to participate into a video conferencing session. After having a participating right confirmation and QoS confirmation, WiMAX xBS sends a request message-JOIN-REQ (WiMAXsSUSIP-Account, Layer Reg) to the ALM group to represent the WiMAXs SU participation in the ALM tree. If the SU node can be added successfully into the ALM tree, an ALM-REP (WiMAXs SU-SIP-Account, UE-ID) response is sent to the WiMAX xBS. WiMAX xBS maps between UE-SIP-Account and WiMAXs SU-ID for managing purposes.

To leave the video conferencing session, WiMAX SUs may send a DeREGISTER(WiMAX-xBS-ID, UE-SIPAccount) or BYE(WiMAX-xBS-ID, WiMAXs SU-SIPAccount) message. In this process, WiMAX-IMS core network will retrieve the reserved resource for the WiMAXs SU. Simultaneously, WiMAX xBS sends leaving request REQ-LEAVE (UE-ID) to ALM tree. The xAS will remove information about the WiMAXs SU from Video conferencing repository. To finish the leaving process, WiMAX xBS will receive the REP-LEAVE(id WiMAXs SU) from the ALM-based overlay group.

\section{C.2 PAUSE/RETURN OPERATION}

Figure 7 shows the Pause/Return operations of a WiMAXs SU over the distributed video conference service. During a video conferencing session, if a WiMAXs 


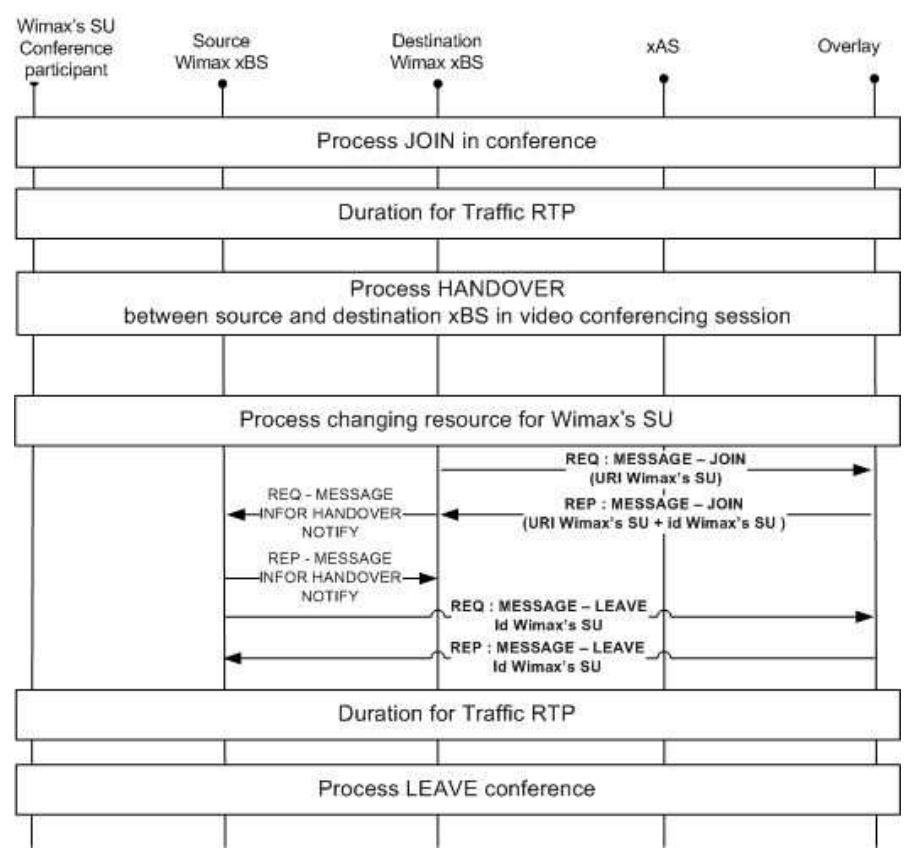

Fig. 8. Hand-over operation when participating in a distributed conference with QoS support.

SU wants to pause the service meaning that the WiMAXs $\mathrm{SU}$ does not want to receive or forward any video data nor signaling messages, but it still wants to return quickly to the ALM group afterwards. To turn into an idle status, WiMAXs SU sends the PAUSE(WiMAXs SUSIP-Account) message to WiMAX xBS, xAS to update theirs status in the repositories. At the same time, WiMAX xBS sends a REQ-PAUSE (WiMAXs SU-ID) to the ALM group. The ALM group answers a REPPAUSE(WiMAXs SU-ID) message to accept the idle status of the WiMAXs SU. After having confirmation from ALM, the IMS-WiMAX core network will change the reserved resource for WiMAXs SU to save network resource. Next, the ALM group stops sending video bit-stream and signaling messages (HEART BEAT message), so that the WiMAXs SU will be stored in the waiting list of the ALM group. After a pre-defined duration, if the WiMAXs SU does not return to video conference service, this ALM group will automatically remove this $\mathrm{SU}$ from the waiting list. If the WiMAX SU wants to return the video conferencing service in a period within the pre-defined duration, it may send a RETURN (WiMAXs SU-Sip-Account, WiMAXs SU-ID) message to WiMAX xBS and xAS. In fact, WiMAX xBS sends a REQ-RETURN (WiMAXs SU-Sip-Account, WiMAXs SU-ID) request to the ALM group. After receiving REP-RETURN (WiMAXs SU-SipAccount, WiMAXs SU-ID) message, the WiMAXs SU can participate in the ALM group. At the time, WiMAXIMS core network will active the reservation resource for WiMAXs SU to rejoin video conferencing session.

\section{C.3 HAND-OVER OPERATION}

Figure 8 presents a WiMAXs SU going through a hand-over process while participating in the distributed

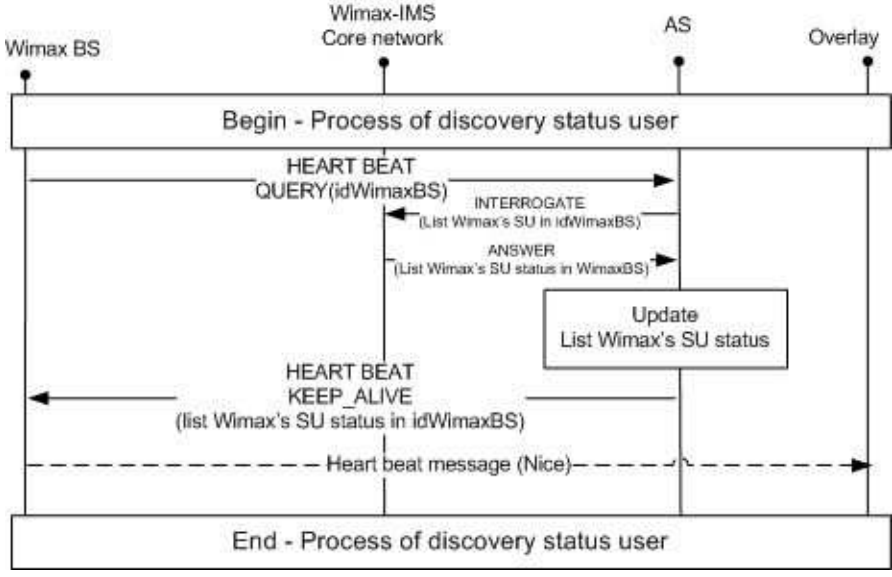

Fig. 9. Hear-beat operation in a distributed conference.

conference. When an UE is about to be handed over from the source to the destination WiMAX xBS, it sends HANDOVER-SUBSCRIBE(Src-WiMAX BS-ID, Des-WiMAX xBS-ID) to the xAS via the source WiMAX xBS. The xAS will then send the HANDOVER-NOTIFY (WiMAXs SU-ID) to the destination WiMAX xBS to notify it about a service hand-over request. A new Resource Reservation process is made for the UE to be attached to the new WiMAX BS. The Destination WiMAX BS then sends a JOIN message to the ALM group to create another UE-ID for the same UE. At this time, two representing nodes are maintained by the two WiMAX XBSs in the ALM group so that a soft hand-over process is obtained. The two of-the-same nodes are sending and receiving bitstreams as any other normal nodes in the ALM group. It will help the UE to maintain its conference service via both WiMAX BSs before the old connection is actually broken. The source WiMAX BS sends a LEAVE-REQ message to the ALM group and waits to receive the LEAVE-REP from the ALM group. Afterwards, the old UE-ID is removed from the ALM group and only one UE-ID will represent the UE in the conference.

\section{C.4 HEARTBEAT OPERATION}

Figure 9 shows the heartbeat handling process in the distributed conference. According to the fact that, many ALM algorithms have to depend on a heartbeat mechanism to maintain their group. If the UE has to directly respond to all heartbeats, it will soon run out of power and computational capacity. Meanwhile, all information regarding a WiMAXs SU availability is available in the core network; a simple interrogation operation triggered by the $\mathrm{xAS}$ can solve this problem. Therefore, the WiMAX xBS will periodically send a HEARTBEAT-QUERY message to the xAS asking for the availability of all WiMAXs SUs which are joining the distributed conference under its representative. When this message is received at the xAS, all WiMAXs SU-IDs are obtained from the Video Conference Repository. Next, an REQ-INTERROGATION (WiMAXs SU-IDs) message containing a list of all WiMAXs SU controlled by the originating WiMAX BS will be sent to the 
WiMAX-IMS Core network. The WiMAX-IMS core will send back the availability information of all requesting UE obtained from the network to the xAS for it to be forwarded to the WiMAX BS. The WiMAX BS will then send back the heartbeats of all the UEs it is representing to the ALM group to inform the group about their status. For floor control purposes, the ALM-based distributed conference will update the list of all peers participating in the conference from the ALM group to the xAS.

\section{Conclusion and Future Work}

Our research proposes the new architecture for internetworking between WiMAX and IMS for a distributed video conferencing service. The advantage of the proposal is to omit the centralized components such as MRFC, MRFP from the standard IMS -based video conferencing architecture. Concurrently, it makes use of WiMAXs BS computational and robust capacity for distributed conferencing service and optimizing mobile network. From the proposal, the new architecture can reduce the signaling and data stream traffic handled by WiMAXs SU when they participate in the distributed video conferencing service.

\section{REFERENCES}

[1] Tien A. Le and Hang Nguyen, "Centralized and distributed architectures of scalable video conferencing services," in Ubiquitous and Future Networks (ICUFN), 2010 Second International Conference on, 2010, pp. 394-399.

[2] Fatna Belqasmi, Chunyan Fu, Mohammed Alrubaye, and Roch Glitho, "Design and implementation of advanced multimedia conferencing applications in the 3GPP IP multimedia subsystem," Comm. Mag., vol. 47, no. 11, pp. 156-163, 2009.

[3] A. Buono, S. Loreto, L. Miniero, and S. P. Romano, "A distributed IMS enabled conferencing architecture on top of a standard centralized conferencing framework [IP Multimedia Systems (IMS) Infrastructure and Services]," Communications Magazine, IEEE, vol. 45, no. 3, pp. 152-159, 2007.

[4] Wimax Forum, "WiMAX Forum Network Architecture - Architecture, detailed Protocols and Procedures - Policy and Charging Control," Tech. Rep., December 2009.

[5] Fangmin $\mathrm{Xu}$, Luyong Zhang, and Zheng Zhou, "Interworking of Wimax and 3GPP networks based on IMS [IP Multimedia Systems (IMS) Infrastructure and Services]," Communications Magazine, IEEE, vol. 45, no. 3, pp. 144-150, 2007.

[6] Suman Banerjee, Bobby Bhattacharjee, and Christopher Kommareddy, "Scalable Application Layer Multicast," 2002.

[7] Stephen E. Deering and David R. Cheriton, "Multicast Routing in a Datagram Internetwork," 1991.

[8] C. Diot, B. N. Levine, B. Lyles, H. Kassem, and D. Balensiefen, "Deployment issues for the IP multicast service and architecture," Network, IEEE, vol. 14, no. 1, pp. 78-88, 2000.

[9] Chong Luo, Wei Wang, Jian Tang, Jun Sun, and Jiang Li, "A Multiparty Video conferencing System Over an Application Level Multicast Protocol," Multimedia, IEEE Transactions on, vol. 9, no. 8, pp. 1621-1632, 2007.

[10] Ming Wu, Fei Wu, and Changsheng Xie, "The Design and Implementation of WiMAX Base Station MAC Based on Intel Network Processor," in Embedded Software and Systems Symposia, 2008. ICESS Symposia '08. International Conference on, 2008. 